\title{
Closed Dressing Chains of 1D and 2D Toda Lattice
}

\author{
Artyom V. Yurov \\ Communicated by Charles Li, received March 1, 2004.
}

\begin{abstract}
We apply the method of dressing chains to reproduction of Toda lattice in the case of $1 \mathrm{D}$ and $2 \mathrm{D}$. On the example of modified equations $m_{0} T L$ and $m_{1} T L$ it is shown that combination of the Darboux and Schlesinger transformations results in closed dressing chains.
\end{abstract}

\section{Contents}

1. Introduction

2. Equations $m_{0} T L$

3. Equations $m_{1} T L$

4. 2D TL equations

5. Conclusion

References

\section{Introduction}

In this work we consider the method of dressing chains of discrete symmetries for the Toda lattice (TL) (including the two-dimensional generalization of them). One starts with nonlinear Schrodinger equation (NLS)

$$
i u_{t}+u_{x x}+2 u^{2} v=-i v_{t}+v_{x x}+2 v^{2} u=0,
$$

which admits two different types of discrete symmetries: Darboux transformation (DT) and Schlesinger transformation (ST). The ST leads to connection between NLS and TL models [1]. Using this circumstance and Lax pair for the NLS model one can find Lax pair for the TL one. Then, using DT for the NLS and Lax pair for the TL one can obtain the DT for the TL. Therefore total theory of TL (Lax pair and DT) can be constructed starting out from the theory of NLS. Using DT for the TL one can construct TL dressing chains to find modified TL equations which

1991 Mathematics Subject Classification. Primary 35; Secondary 34.

Key words and phrases. Dressing chains, Toda lattice, Darboux transformation, Schlesinger transformation. 
will be denoted as $m^{1} T L$ (with superscript). To do that it is enough to know $L$ operator of the NLS. By repeating this procedure with $A$-operator of the equation (1) one find symmetries of the $m^{1} T L$ (the exact definition of these symmetries is contained in [2]). This plan is realized in the Section 2. We'll show that it is appears the closed dressing chains which tie together TL and Volterra equations, in contrast to open-ended dressing chains of the KdV and NLS equations. This is the character of the dressing chains of the TL equations.

The alternative path to "multiply" the Toda lattice (see Sec. 3) is as follows: One construct discrete symmetry chain for the NLS to find modified NLS equations (we denote them by the $m_{k} N L S$ ). Each of them inherits a ST from an initial NLS (1) which leads to corresponding Toda-like lattice equations - $m_{k} T L$ (with subscript). Since Lax pair and Darboux transformations for the $m_{k} N L S$ are known (this is crucial point of the dressing chains method) then one can find Lax pairs and DT for the $m_{k} T L$. Now, if we apply the method from the previous paragraph then one will find a new family of Toda-like equations (we denote them by the as $m_{k}^{n} T L$. The subscript points to the number of the modified NLS which creates this $m_{k}^{n} T L$ equation. For example, all Toda-like equations from the Sec. 2 belong to class $m_{0} T L$, while all ones from the Sec. 3 belong to class $\left.m_{1} T L\right)$.

In Sec. 4 we generalize our approach to $2 D$ TL models using the DavyStewartson equations (DS). Dressing chains of the first and second types are constructed.

There are many works which are devoted to links between integrable NLS-like models and difference-differential Toda-like lattices (see, for example, [3]). The classification of integrable lattice was made in [2], [4]. Our results are associated with results in [5] and we are discussing that in Sec. 5. This work is continuation of our research in [6], [7] which, for one's turn, was inspired by the article [8].

\section{Equations $m_{0} T L$}

Lax pair for the (1) has the form

$$
\Psi_{x}=-i \sigma_{3} \Psi \Lambda+i S \Psi, \quad \Psi_{t}=-2 i \sigma_{3} \Psi \Lambda^{2}+2 i S \Psi \Lambda+W \Psi,
$$

where

$$
S=\left(\begin{array}{cc}
0 & u \\
v & 0
\end{array}\right), \quad \Lambda=\left(\begin{array}{cc}
\lambda & 0 \\
0 & \mu
\end{array}\right), \quad W=\sigma_{3}\left(i S^{2}-S_{x}\right),
$$

$\lambda$ and $\mu$ are spectral parameters, $\sigma_{3}$ is the third Pauli matrix, $\Psi-2 \times 2$ matrix function. We denote componentry of the first matrix $\Psi$ column by $\psi_{n}, \phi_{n}, u \equiv u_{n}$, $v \equiv v_{n}$. Then, the L-equation of the system (2) has the form

$$
\psi_{n, x}=-i \lambda \psi_{n}+i u_{n} \phi_{n}, \quad \phi_{n, x}=i \lambda \phi_{n}+i v_{n} \psi_{n} . \quad(3 a-b)
$$

The components of the second $\Psi$ column are satisfying the similar system with substitution $\lambda \rightarrow \mu$.

The ST for the (1) are

$$
\begin{gathered}
u_{n} \rightarrow u_{n+1}=u_{n}\left[u_{n} v_{v}+\left(\log u_{n}\right)_{x x}\right], \quad v_{n} \rightarrow v_{n+1}=\frac{1}{u_{n}}, \quad(4 a-b) \\
\psi_{n} \rightarrow \psi_{n+1}=\left(-2 \lambda+i\left(\log u_{n}\right)_{x}\right) \psi_{n}+u_{n} \phi_{n}, \quad \phi_{n} \rightarrow \phi_{n+1}=\frac{\psi_{n}}{u_{n}}, \quad(5 a-b)
\end{gathered}
$$


and reverse conversion:

$$
\begin{aligned}
& v_{n} \rightarrow v_{n-1}=v_{n}\left[u_{n} v_{v}+\left(\log v_{n}\right)_{x x}\right], \quad u_{n} \rightarrow u_{n-1}=\frac{1}{v_{n}}, \\
& \phi_{n} \rightarrow \phi_{n-1}=\left(2 \lambda+i\left(\log v_{n}\right)_{x}\right) \phi_{n}+v_{n} \psi_{n}, \quad \psi_{n} \rightarrow \psi_{n-1}=\frac{\phi_{n}}{v_{n}} .
\end{aligned}
$$

Let us denote

$$
q_{n} \equiv \log \left(u_{n}\right), \quad p_{n} \equiv q_{n, x}, \quad U_{n} \equiv \frac{u_{n}}{u_{n-1}}=e^{q_{n}-q_{n-1}} .
$$

It is easy to examine that functions $q_{n}$ are solutions of the TL equations

$$
q_{n, x x}=e^{q_{n+1}-q_{n}}-e^{q_{n}-q_{n-1}} .
$$

This remarkable point was noted in many works (see, for example, [1]-[3]). Our first aim here is to construct Lax pair for the (8) starting out from the equations (3). It is comfortably to introduce the shift-operator $T$ :

$$
T u_{n}=u_{n+1} \text {. }
$$

We act $T^{-1}$ on the equation (5b) then substitute the expression for the $\phi_{n}$ into (3a) to obtain the first equation of the Lax pair:

$$
\psi_{n, x}=-i \lambda \psi_{n}+i U_{n} \psi_{n-1} .
$$

To obtain the second equation of the Lax pair we act $T$ onto the (3b) and substitute into this expression $\phi_{n+1}$ (from the (5b)), $v_{n+1}$ (from the (4b)) and $\psi_{n, x}$ from the (9). As a result one get

$$
\psi_{n+1}=\left(2 \lambda+i p_{n}\right) \psi_{n}+U_{n} \psi_{n-1} .
$$

The consistency constraints of (9) and (10) has the form of two equations

$$
p_{n, x}=U_{n+1}-U_{n}, \quad U_{n, x}=U_{n}\left(p_{n}-p_{n-1}\right),
$$

which can be transformed into the (8) by the (7). Thus, (9) and (10) are nothing but Lax pair for the TL model.

Let us construct DT for the TL equation using elementary DT for the NLS model (1) (see [9]). Let $\psi_{1}$ and $\phi_{1}$ be the components of the first matrix $\Psi$ column, where $\Psi$ is the solution of the (2) with $\lambda=\lambda_{1}$. Then one can write two sorts of the DT (the indices are omitted):

$$
\begin{aligned}
& \psi \rightarrow \psi^{(1)}=\left[2\left(\lambda-\lambda_{1}\right)+\frac{\phi_{1}}{\psi_{1}} u\right] \psi-u \phi, \quad \phi \rightarrow \phi^{(1)}=\phi-\frac{\phi_{1}}{\psi_{1}} \psi, \\
& u \rightarrow u^{(1)}=i u_{x}-\left(2 \lambda_{1}-\frac{\phi_{1}}{\psi_{1}} u\right) u, \quad v \rightarrow v^{(1)}=\frac{\phi_{1}}{\psi_{1}}
\end{aligned}
$$

and

$$
\begin{aligned}
& \psi \rightarrow{ }^{(1)} \psi=\psi-\frac{\psi_{1}}{\phi_{1}} \phi, \quad \phi \rightarrow{ }^{(1)} \phi=\left[2\left(\lambda_{1}-\lambda\right)+\frac{\psi_{1}}{\phi_{1}} v\right] \phi-v \psi, \\
& u \rightarrow{ }^{(1)} u=\frac{\psi_{1}}{\phi_{1}}, \quad v \rightarrow{ }^{(1)} v=i v_{x}+\left(2 \lambda_{1}+\frac{\psi_{1}}{\phi_{1}} v\right) v .
\end{aligned}
$$

Let's $\psi_{2}, \phi_{2}$ be components of the second matrix $\Psi$ column with $\mu=\lambda_{2}$. Then one make transformations (11) and (12):

$$
u \rightarrow u^{(1)} \rightarrow{ }^{(2)} u^{(1)}, \quad v \rightarrow v^{(1)} \rightarrow{ }^{(2)} v^{(1)},
$$




$$
\psi \rightarrow \psi^{(1)} \rightarrow^{(2)} \psi^{(1)}, \quad \phi \rightarrow \phi^{(1)} \rightarrow^{(2)} \phi^{(1)} .
$$

Note that the DT transformations are commutating

$$
{ }^{(2)} u^{(1)}={ }^{(1)} u^{(2)}, \quad{ }^{(2)} v^{(1)}={ }^{(1)} v^{(2)} .
$$

If we'll write (13) explicitly for the twice dressed potentials and wave functions it is easy to see that we would obtain the "ordinary" DT for the NLS (see [10]). This is why we can call (11) and (12) as elemantary DTs. Unfortunately these transformations are uncomfortable in sense of getting exact solutions of the NLS because, in the case of general position, it is impossible to conserve reduction restriction $u= \pm v^{*}$, in contrast to (13).

Now it is easy to find DT for the TL equations. Omitting simple calculations we'll show the result. Let $\left\{\psi_{1, n}\right\}$ be the solution of the Lax pair (9), (10) with $\lambda=\lambda_{1}$. Then we have two DTs for the TL equations which will be referred to as $R$-transformation and $L$-transformation:

$$
R: \quad \psi_{n} \rightarrow \psi_{n}^{(1)}=-\psi_{n+1}+\frac{\psi_{1, n+1}}{\psi_{1, n}} \psi_{n}, \quad q_{n} \rightarrow q_{n}^{(1)}=q_{n}+\log \frac{\psi_{1, n+1}}{\psi_{1, n}}
$$

and

$$
L: \quad \psi_{n} \rightarrow{ }^{(1)} \psi_{n}=\psi_{n}-\frac{\psi_{1, n}}{\psi_{1, n-1}} \psi_{n-1}, \quad q_{n} \rightarrow{ }^{(1)} q_{n}=q_{n-1}+\log \frac{\psi_{1, n}}{\psi_{1, n-1}} .
$$

Using (9)-(10) it is quit easy to find first modified TL equations. For this purpose one introduce new functions $\tau_{n}$ and $\xi_{n}$

$$
\tau_{n}=\frac{\psi_{1, n}}{\psi_{1, n+1}}=\frac{1}{\partial \xi_{n}}
$$

where $\partial \equiv-i \partial / \partial x, V_{n} \equiv i p_{n}$. The shape of the function $\tau_{n}$ is dictated from the (14) and (15). In new dependent variables, the Lax pair for the TL equations has the form:

$$
\partial \tau_{n}=\tau_{n}\left(\tau_{n-1} U_{n}-\tau_{n} U_{n+1}\right), \quad \tau_{n+1}=\frac{1}{2 \lambda_{1}+V_{n+1}+\tau_{n} U_{n+1}} .
$$

Note that the first equations has quadratic nonlinearity by fields $\tau_{n}$, as it should be in the method of the dressing chains ([8]).

Eliminating potentials $U_{n}, U_{n+1}$ and $V_{n+1}$ from the (16) one get equation $m^{1} T L$, which is just a Volterra equation (one can write this in more customary form; see (17a), (52)):

$$
\partial^{2} \xi_{n}=\partial \xi_{n}\left(e^{\xi_{n}-\xi_{n+1}}-e^{\xi_{n-1}-\xi_{n}}\right) .
$$

To obtain dressing chains of discrete symmetries one can use either $R$ or $L$ transformations (14), (15). Let us choose the (14). At the same time, the transformations regulation for the $U_{n}$ and $V_{n}$ has the form:

$$
U_{n} \rightarrow U_{n}^{(1)}=\frac{\tau_{n-1}}{\tau_{n}} U_{n}, \quad V_{n} \rightarrow V_{n}^{(1)}=V_{n}+\tau_{n-1} U_{n}-\tau_{n} U_{n+1}
$$

Let's take $\psi_{2, n}$ as the solution of (9), (10) with $\lambda=\lambda_{2}, \psi_{2, n}^{(1)}$ is calculated from the (14) whereas $\zeta_{n}$ is defined by the expression:

$$
\partial \zeta_{n}=\frac{\psi_{2, n+1}^{(1)}}{\psi_{2, n}^{(1)}}
$$


After simple calculations one get these chains:

$$
\begin{gathered}
e^{\zeta_{n}-\zeta_{n+1}} \partial \zeta_{n}=e^{\xi_{n}-\xi_{n+1}} \partial \xi_{n+1}, \\
\partial\left(\zeta_{n}-\xi_{n}\right)=2\left(\lambda_{2}-\lambda_{1}\right)+e^{\zeta_{n-1}-\zeta_{n}}-e^{\xi_{n}-\xi_{n+1}} .
\end{gathered}
$$

(18) contain Lax pair for the (17). Denoting $\zeta_{n}=\log \Psi_{n}$, we get

$$
\partial \Psi_{n}=A_{n} \Psi_{n+1}=\left(B_{n-1}+\tilde{\lambda}\right) \Psi_{n}+\Psi_{n-1},
$$

where

$$
A_{n}=e^{\xi_{n}-\xi_{n+1}} \partial \xi_{n+1}, \quad B_{n}=\partial \xi_{n+1}-e^{\xi_{n+1}-\xi_{n+2}},
$$

and new spectral parameter $\tilde{\lambda}$ is connected with the $\lambda$ by the proportion: $\tilde{\lambda}=$ $2\left(\lambda-\lambda_{1}\right)$. We note that the consistency condition of (19) has the form of usual Toda lattice

$$
\partial A_{n}=A_{n}\left(B_{n-1}-B_{n}\right), \quad \partial B_{n}=A_{n+1}-A_{n}
$$

and can be reduced to the (17) by the substitution (20) into the (21). In new dependent variable the $R$-transformation (14) is nothing else but $L$-transformation (15). In other words, one obtain the closed chains of discrete symmetries which connect TL equations with Volterra equations $\left(m^{1} T L\right)$.

To complete the picture we'll cite the corresponding formulas. Let $\Psi_{1, n}$ be the solution of (19) with $\tilde{\lambda}=\tilde{\lambda}_{1} \neq 0 . R$-transformation (14) induce $L$-transformation (15) for the (19) and (21) (one can examine it by the direct calculation ):

$$
\begin{aligned}
& \Psi_{n} \rightarrow^{(1)} \Psi_{n}=\Psi_{n}-\sigma_{n} \Psi_{n+1}, \quad A_{n} \rightarrow^{(1)} A_{n}=A_{n}-\partial \sigma_{n}, \\
& B_{n} \rightarrow^{(1)} B_{n}=B_{n+1}+\partial \log \sigma_{n+1},
\end{aligned}
$$

where $\sigma_{n}=\Psi_{1, n} / \Psi_{1, n+1}$ are solutions of system (compareto (16)):

$$
\partial \sigma_{n}=A_{n}-\left(B_{n}+\tilde{\lambda}_{1}\right) \sigma_{n}-\sigma_{n}^{2}, \quad \sigma_{n+1}=\frac{A_{n+1}}{B_{n}+\sigma_{n}+\tilde{\lambda}_{1}} .
$$

Using (21) and (22) we eliminate potentials $B_{n}$ and again obtain Volterra equations:

$$
\partial \log \sigma_{n}=\beta_{n}-\beta_{n+1}, \quad \partial \log \beta_{n}=\sigma_{n}-\sigma_{n-1},
$$

where $\beta_{n}=A_{n} / \sigma_{n}$.

Analogously, one can consider t-equations. Rewriting (1) in new variables $q_{n}$, $p_{n}$, and taking (8) into account we get the known symmetry of the equations (8):

$$
-i q_{n, t}=p_{n}^{2}+e^{q_{n+1}-q_{n}}+e^{q_{n}-q_{n-1}} .
$$

It is possible to repeat all actions described above: to find Lax pair, then to apply Darboux transformation to construct symmetry of the Volterra equations. Resulting formulas has been described before (with the help of another approach; see [2], [4]) so we omit them here. Note that [2] contains the total (up to gauge, linear, Galilean transformations) list of integrable generalization of classical and relativist Toda lattice in the form

$$
q_{n, x x}=\frac{1}{2}\left(G\left(q_{n+1}, q_{n}, p_{n+1}, p_{n}\right)-F\left(q_{n}, q_{n-1}, p_{n}, p_{n-1}\right)\right),
$$

with symmetries

$$
q_{n, t}=\frac{1}{2}\left(G\left(q_{n+1}, q_{n}, p_{n+1}, p_{n}\right)+F\left(q_{n}, q_{n-1}, p_{n}, p_{n-1}\right)\right) .
$$


Using the method of dressing chains we have found only two equations from this list. In the next Section we'll obtain new generalization of TL equations.

\section{Equations $m_{1} T L$}

Using DT (13) and Lax pair for the NLS (1) we can construct the first modified NLS $\left(m_{1} N L S\right)$ which has the form

$$
\begin{gathered}
\left(\beta^{2}-4 b c\right)\left(i b_{t}+b_{x x}-2 b^{2} c\right)+2 \alpha\left(\alpha c+2 i c_{x}\right) b^{2}+2\left(b_{x} c+2 b c_{x}\right) b_{x}=0, \\
\left(\beta^{2}-4 b c\right)\left(-i c_{t}+c_{x x}-2 c^{2} b\right)+2 \alpha\left(\alpha b-2 i b_{x}\right) c^{2}+2\left(b c_{x}+2 b_{x} c\right) c_{x}=0,
\end{gathered}
$$

where $\alpha=\lambda+\mu, \beta=\lambda-\mu, \lambda$ and $\mu$ are spectral parameters of (2) as before, and $b=b(x, t)$ with $c=c(x, t)$ are new dependent variables which are defined by

$$
b=\frac{u-{ }^{(2)} u^{(1)}}{2}, \quad c=\frac{{ }^{(2)} v^{(1)}-v}{2} .
$$

If $\alpha=\beta=0$, then the equation (24) take the elegant form

$$
i b_{t}+b_{x x}-2 b^{2} c-\frac{b_{x} c_{x}}{c}-\frac{1}{2} \frac{b_{x}^{2}}{b}=0, \quad-i c_{t}+c_{x x}-2 b c^{2}-\frac{b_{x} c_{x}}{b}-\frac{1}{2} \frac{c_{x}^{2}}{c}=0 .
$$

STs for the (25) are defined by the formulas (compare to(4), (6)):

$$
\begin{aligned}
b_{n} \rightarrow b_{n+1} & =\frac{1}{4} \frac{\left(2 c_{n} b_{n, x}^{2}-2 c_{n} b_{n} b_{n, x x}+c_{n, x} b_{n, x} b_{n}+4 c_{n}^{2} b_{n}^{3}\right)^{2}}{c_{n}^{2} b_{n}\left(b_{n, x}^{2}-4 c_{n} b_{n}^{3}\right)}, \\
c_{n} \rightarrow c_{n+1} & =\frac{4 c_{n} b_{n}^{2}}{b_{n, x}^{2}-4 c_{n} b_{n}^{3}}, \quad b_{n} \rightarrow b_{n-1}=\frac{4 b_{n} c_{n}^{2}}{c_{n, x}^{2}-4 b_{n} c_{n}^{3}}, \\
c_{n} \rightarrow c_{n-1} & =\frac{1}{4} \frac{\left(2 b_{n} c_{n, x}^{2}-2 b_{n} c_{n} c_{n, x x}+b_{n, x} c_{n, x} c_{n}+4 b_{n}^{2} c_{n}^{3}\right)^{2}}{b_{n}^{2} c_{n}\left(c_{n, x}^{2}-4 b_{n} c_{n}^{3}\right)},
\end{aligned}
$$

where

$$
\left(b_{n+1}\right)_{n-1}=\left(b_{n-1}\right)_{n+1}=b, \quad\left(c_{n+1}\right)_{n-1}=\left(c_{n-1}\right)_{n+1}=c .
$$

Formulas (26) were obtained from the discrete symmetries chains of NLS in our work [7]. There is another (more spontaneous) way to obtain them. This way is based on the comparative analysis of (25) and (1). Let's consider (for distinctness) the transformation $n \rightarrow n+1$ in (26). In the case of ST (4a-b) for the NLS one can see that new fields are expressed through old ones via formulas (most of indices are omitted in order to prevent jamming of formulas):

$$
u_{n+1}=U\left(u, v, u_{x}, u_{x x}\right), \quad v_{n+1}=V(u) .
$$

By analogy with (27), one need pick out for the (25)

$$
b_{n+1}=B(b, c, q, p, w), \quad c_{n+1}=C(b, c, q),
$$

where values $q=b_{x}, p=c_{x}, w=q_{x}$ must be considered as independent variables whereas $B$ and $C$ should be obtained. Now we should substitute (28) into mNLS then eliminate time derivatives with the help of (25) and, at the end of this, to equate to zero expessions attached to independent variables. In such a way, equating to zero the factor attached to $w_{x}$ in the second equation (25) we get (after simple integration by $w)$ :

$$
B=\left(w C_{q}+q C_{b}+p C_{c}\right)^{2} S(b, c, q, p),
$$


where $S(b, c, q, p)$ is arbitrary function of it's arguments. By equating to zero the factor attached to $w$ one get

$$
C_{q}=0, \quad S_{p}=0 .
$$

The further inquiry show that we need to choose the second way so $S=Z(b, c, q)$. Then, the nulling of the factor attached to $p_{x}$ results in simple PDE and the integration of this equation gives us the functional dependence for the $C$ :

$$
C=C\left(b, \frac{q^{2}}{c}\right) .
$$

The factor attached to $w^{2}$ leads to Riccati equation (that's a bad news) but we can simplify the problem (here are a good ones) using the following observation: Let

$$
b=g_{1}(t)+\epsilon f_{1}(x, t), \quad c=g_{2}(t)+\epsilon f_{2}(x, t) .
$$

Substituting (29) into the (25) we see that if $\epsilon \rightarrow 0$ then two last items in (25) are negligible quantities so the equations (25) are reduced to (non-soliton) NLS. This implies that if $\epsilon \rightarrow 0$ then our desired ST must be reduced into ST (4). Therefore

$$
\frac{1}{C}=-b+F\left(b, \frac{q^{2}}{c}\right),
$$

where $F(b, 0)=0(\epsilon \rightarrow 0$ mean that $q \rightarrow 0$ and $p \rightarrow 0)$. The last condition means that for the $F$ one can use Taylor with respect to $q^{2} / c$. Since the dimensionalities of $b, c$ and $x$ are connected by the proportion $[b][c]=[x]^{-2}$, we get the initial object for the $C$ :

$$
\frac{1}{C}=-b+\sum_{m=1}^{\infty} G_{m}\left(\frac{q^{2}}{c}\right)^{m} b^{1-3 m},
$$

where $G_{m}$ are dimensionless constants. In fact, it is enough to restrict ourself to the first term of series in (30) (we can verify, comparing (30) with (26), that $G_{1}=1 / 4$ whereas the rest coefficients are zero). Using this obtained initial object one can continue the calculation to find (26).

If $\alpha$ and $\beta$ are nonvanishing numbers then

$$
\begin{aligned}
b_{n-1} & =\frac{i c_{n}\left(\beta^{2}-4 b_{n} c_{n}\right)}{i\left(\alpha^{2}-\beta^{2}\right) c_{n}^{2}-2 \alpha c_{n} c_{n, x}+i\left(4 c_{n}^{3} b_{n}-c_{n, x}^{2}\right)}, \\
c_{n-1} & =\frac{Z\left(-\alpha, \beta, c_{n}, b_{n}\right)}{\left(\beta^{2}-4 b c\right)^{2}\left[i\left(\alpha^{2}-\beta^{2}\right) c_{n}^{2}-2 \alpha c_{n} c_{n, x}+i\left(4 c_{n}^{3} b_{n}-c_{n, x}^{2}\right)\right]}, \\
b_{n+1} & =\frac{Z\left(\alpha, \beta, b_{n}, c_{n}\right)}{\left(\beta^{2}-4 b c\right)^{2}\left[i\left(\alpha^{2}-\beta^{2}\right) b_{n}^{2}+2 \alpha b_{n} b_{n, x}+i\left(4 b_{n}^{3} c_{n}-b_{n, x}^{2}\right)\right]}, \\
c_{n+1} & =\frac{i b_{n}\left(\beta^{2}-4 b_{n} c_{n}\right)}{i\left(\alpha^{2}-\beta^{2}\right) b_{n}^{2}+2 \alpha b_{n} b_{n, x}+i\left(4 b_{n}^{3} c_{n}-b_{n, x}^{2}\right)} .
\end{aligned}
$$

The quantity $Z(\alpha, \beta, b, c)$ has bulky form so we'll show it in two extreme cases when either $\beta=0$ or $\alpha=0$ :

$$
\begin{aligned}
\frac{Z(\alpha, 0, b, c)}{4 b}= & -i\left(b^{2} c \alpha^{2}\right)^{2}+2 b^{3} c\left(b c_{x}-b_{x} c\right) \alpha^{3}-i b^{2}\left[3\left(b_{x} c\right)^{2}+(3 b c)^{3}\right. \\
& \left.+4 b c\left(b_{x} c_{x}-c b_{x x}\right)-\left(b c_{x}\right)^{2}\right] \alpha^{2}+2 b\left(b c_{x}-b_{x} c\right)\left((2 c b)^{2} b+b_{x} c_{x} b\right. \\
& \left.-2 b c b_{x x}+2 b_{x}^{2} c\right) \alpha-i\left((2 c b)^{2} b+b_{x} c_{x} b-2 b c b_{x x}+2 b_{x}^{2} c\right)^{2}
\end{aligned}
$$




$$
\begin{aligned}
-i Z(0, \beta, b, c)= & b\left(b^{3} c+b_{x}^{2}-b b_{x x}\right) \beta^{6}+\left[\left(b_{x}^{2}-b b_{x x}\right) b_{x x}-2 b^{3} b_{x} c_{x}\right. \\
& \left.-12 b^{2} c\left(b^{3} c+b_{x}^{2}-b b_{x x}\right)\right] \beta^{4}+\left[48 b^{3} c^{2}\left(b_{x}^{2}+b^{3} c-b b_{x x}\right)\right. \\
& +8 c\left(b b_{x x}\right)^{2}-4 b b_{x}\left(3 b_{x} c+b c_{x}\right) b_{x x}+\left(3 c b_{x}^{2}+2 b c_{x} b_{x}^{2}\right. \\
& \left.\left.+16 b^{4} c c_{x}\right) b_{x}\right] \beta^{2}-4 b\left((2 c b)^{2} b+b_{x} c_{x} b-2 b c b_{x x}+2 b_{x}^{2} c\right)^{2} .
\end{aligned}
$$

Formulas (31), (32) result in new Toda-like lattice. Denoting

$$
P_{n}=\log b_{n}, \quad Q_{n}=\log c_{n},
$$

one find the equations $m_{1}^{0} T L \equiv m_{1} T L$ in familiar Euclidean variables:

$$
\begin{aligned}
& \left(\partial P_{n}\right)^{2}+2 \alpha \partial P_{n}+4 e^{P_{n}+Q_{n}}-\beta^{2} e^{-P_{n}-Q_{n+1}}+4 e^{Q_{n}-Q_{n+1}}=\beta^{2}-\alpha^{2}, \\
& \left(\partial Q_{n}\right)^{2}-2 \alpha \partial Q_{n}+4 e^{P_{n}+Q_{n}}-\beta^{2} e^{-Q_{n}-P_{n-1}}+4 e^{P_{n}-P_{n-1}}=\beta^{2}-\alpha^{2} .
\end{aligned}
$$

Thus we have integrable lattice composed of interaction nodes (atoms) of two kinds. It is most simple case when $\alpha=\beta=0$. In this case each equation looks as a law of each atom's total energy conservation. In other words, in this case the equations (33) describes zero-point oscillations of lattice.

Lax pair for the (33) can be obtained via NLS discrete symmetries chains. As a result one get

$$
\begin{aligned}
& \left(\alpha-2 a_{n}\right) \partial \Psi_{n}+2\left(\alpha_{1}-A_{n}\right)\left(\alpha-2 a_{n}\right) \Psi_{n}-\left(\alpha_{1}-2 A_{n}\right)\left(\partial b_{n}+2 a_{n} b_{n}\right)=0, \\
& \Psi_{n-1}=\frac{\left(\beta_{1}^{2}-4 \Psi_{n} \Phi_{n}\right) \Phi_{n}}{\left(\alpha_{1}^{2}-\beta_{1}^{2}\right) \Phi_{n}^{2}-2 \alpha_{1} \Phi_{n} \partial \Phi_{n}+4 \Phi_{n}^{3} \Psi_{n}+\left(\partial \Phi_{n}\right)^{2}}, \\
& \left(\alpha-2 a_{n}\right) \partial \Phi_{n}+2\left(\left(\alpha-a_{n}\right) \alpha_{1}+\left(4 a_{n}-3 \alpha\right) A_{n}\right) \Phi_{n}-\left(\alpha-2 A_{n}\right) \partial c_{n}=0, \\
& \Phi_{n+1}=\frac{\left(\beta_{1}^{2}-4 \Psi_{n} \Phi_{n}\right) \Psi_{n}}{\left(\alpha_{1}^{2}-\beta_{1}^{2}\right) \Psi_{n}^{2}+2 \alpha_{1} \Psi_{n} \partial \Psi_{n}+4 \Psi_{n}^{3} \Phi_{n}+\left(\partial \Psi_{n}\right)^{2}},
\end{aligned}
$$

where $\Psi_{n}, \Phi_{n}$ are wave functions of the spectral problem, $\alpha_{1}, \beta_{1}$ are spectral parameters ( $\alpha$ and $\beta$ are fixed) whereas functions $a_{n}, A_{n}$ are defined as:

$$
a_{n}=\frac{1}{2}\left(\alpha \pm \sqrt{\beta^{2}-4 b_{n} c_{n}}\right), \quad A_{n}=\frac{1}{2}\left(\alpha_{1} \pm \sqrt{\beta_{1}^{2}-4 \Psi_{n} \Phi_{n}}\right) .
$$

Equations (33), (34) are analogue of Lax pair (16) for the TL equations (8). It is quite obvious from (34) that $\log \Psi_{n}, \log \Phi_{n}$ are solutions of the same $m_{1} T L$ equations (33) with the change $\alpha \rightarrow \alpha_{1}, \beta \rightarrow \beta_{1}$.

At last, using (13) it is easy to calculate corresponding Darboux transformation for the (34). One can predict the response:

$$
b_{n} \rightarrow \Psi_{n}, \quad c_{n} \rightarrow \Phi_{n} .
$$

Intermediate equations $m_{1}^{2} T L$ (i.e. the analogue of the Volterra equation for the (33)) can be obtained from the above mentioned formulas:

$$
\hat{\Psi}_{n}=\Psi_{n}-b_{n}, \quad \hat{\Phi}_{n}=\Phi_{n}-c_{n} .
$$

The Lax pair for the $m_{1}^{2} T L$ equation can be obtained from the (34) by the elimination of $\Psi_{n}$ and $\Phi_{n}$ with the help of the (35). In the role of wave function one get $b_{n}$ and $c_{n}$ (with another values of $\alpha, \beta$ ). Thus, we get closed dressing chains again. 


\section{2D TL equations}

Above-stated formalism is also work for the $2 D$ TL equations. These equations can be obtained from the DS equations:

$$
\begin{gathered}
i u_{t}+u_{x x}+\frac{1}{\alpha^{2}} u_{y y}-\frac{2}{\alpha^{2}} u^{2} v+g u=0, \\
-i v_{t}+v_{x x}+\frac{1}{\alpha^{2}} v_{y y}-\frac{2}{\alpha^{2}} v^{2} u+g v=0, \quad g_{y y}-\alpha^{2} g_{x x}=-4(u v)_{x x} .
\end{gathered}
$$

Here $\alpha^{2}= \pm 1$. The Lax "pair" for the (36) is the system of four scalar equations,

$$
\begin{aligned}
& \psi_{y}=\alpha \psi_{x}+u \phi, \quad \phi_{y}=-\alpha \phi_{x}+v \psi, \\
& \psi_{t}=2 i \psi_{x x}+\frac{2 i}{\alpha} u \phi_{x}+\left(\frac{1}{2}\left[\frac{1}{\alpha} F_{y}+F_{x}\right]-\frac{i}{\alpha^{2}} u v\right) \psi+\frac{i}{\alpha^{2}}\left(\alpha u_{x}+u_{y}\right) \phi, \\
& \phi_{t}=-2 i \phi_{x x}+\frac{2 i}{\alpha} v \psi_{x}+\left(\frac{1}{2}\left[\frac{1}{\alpha} F_{y}-F_{x}\right]+\frac{i}{\alpha^{2}} u v\right) \phi+\frac{i}{\alpha^{2}}\left(\alpha v_{x}-v_{y}\right) \psi,
\end{aligned}
$$

where $g=-i F_{x}$.

Now let two twains of functions $\left\{\psi_{1}, \phi_{1} ; \psi, \phi\right\}$ be solution of the (37) with any given $u, v$ and $F$. Elementary Darboux transformations for the DS equations (i.e. the analogue of the (11), (12)) has the form,

$$
\begin{aligned}
& \psi \rightarrow \psi^{(1)}=-2 \alpha \psi_{x}-u \phi+\left(2 \alpha \psi_{1, x}+u \phi_{1}\right) \frac{\psi}{\psi_{1}}, \quad \phi \rightarrow \phi^{(1)}=\phi-\frac{\phi_{1}}{\psi_{1}} \psi, \\
& u \rightarrow u^{(1)}=\frac{u}{\psi_{1}}\left(2 \alpha \psi_{1, x}+u \phi_{1}\right)-u_{y}-\alpha u_{x}, \quad v \rightarrow v^{(1)}=\frac{\phi_{1}}{\psi_{1}}, \\
& F \rightarrow F^{(1)}=F+4 i \frac{\psi_{1, x}}{\psi_{1}}
\end{aligned}
$$

and

$$
\begin{aligned}
& \phi \rightarrow^{(1)} \phi=2 \alpha \phi_{x}-v \psi-\left(2 \alpha \phi_{1, x}-v \psi_{1}\right) \frac{\phi}{\phi_{1}}, \quad \psi \rightarrow^{(1)} \psi=\psi-\frac{\psi_{1}}{\phi_{1}} \phi, \\
& v \rightarrow^{(1)} v=\frac{v}{\phi_{1}}\left(v \psi_{1}-2 \alpha \phi_{1, x}\right)-v_{y}+\alpha v_{x}, \quad u \rightarrow^{(1)} u=\frac{\psi_{1}}{\phi_{1}}, \\
& F \rightarrow{ }^{(1)} F=F+4 i \frac{\phi_{1, x}}{\phi_{1}} .
\end{aligned}
$$

Two-dimensional problem has larger list of discrete symmetries then single-dimensional one. The new type of them is so called binary DT which can be obtained as follows: DS equations has Lax pair which is "conjugate" to (37),

$$
\begin{aligned}
& p_{y}=\alpha p_{x}-v f, \quad f_{y}=-\alpha f_{x}-u p, \\
& p_{t}=-2 i p_{x x}+\frac{2 i}{\alpha} v f_{x}-\left(\frac{1}{2}\left[\frac{1}{\alpha} F_{y}+F_{x}\right]-\frac{i}{\alpha^{2}} u v\right) p+\frac{i}{\alpha^{2}}\left(\alpha v_{x}+v_{y}\right) f, \\
& f_{t}=2 i f_{x x}+\frac{2 i}{\alpha} u p_{x}-\left(\frac{1}{2}\left[\frac{1}{\alpha} F_{y}-F_{x}\right]+\frac{i}{\alpha^{2}} u v\right) f+\frac{i}{\alpha^{2}}\left(\alpha u_{x}-u_{y}\right) p .
\end{aligned}
$$


For accommodation we denote wave function of Lax pair (40) using Roman alphabet $p, f$ (Greek letters $\psi, \phi$ will be used for the Lax pair (37)), and DT for the (40) will be marked with the help of subscripts in round paranthesis:

$$
\begin{aligned}
& p \rightarrow p_{(1)}=2 \alpha p_{x}-v f-\left(2 \alpha p_{1, x}-v f_{1}\right) \frac{p}{p_{1}}, \quad f \rightarrow f_{(1)}=f-\frac{f_{1}}{p_{1}} p, \\
& v \rightarrow v_{(1)}=\frac{v}{p_{1}}\left(v f_{1}-2 \alpha p_{1, x}\right)+v_{y}+\alpha v_{x}, \quad u \rightarrow u_{(1)}=\frac{f_{1}}{p_{1}}, \\
& F \rightarrow F_{(1)}=F+4 i \frac{p_{1, x}}{p_{1}}, \\
& f \rightarrow_{(1)} f=-2 \alpha f_{x}-u p+\left(2 \alpha f_{1, x}+u p_{1}\right) \frac{f}{f_{1}}, \quad p \rightarrow_{(1)} p=p-\frac{p_{1}}{f_{1}} f, \\
& u \rightarrow{ }_{(1)} u=\frac{u}{f_{1}}\left(u p_{1}+2 \alpha f_{1, x}\right)+u_{y}-\alpha u_{x}, \quad v \rightarrow_{(1)} v=\frac{p_{1}}{f_{1}}, \\
& F \rightarrow{ }_{(1)} F=F+4 i \frac{f_{1, x}}{f_{1}} .
\end{aligned}
$$

DT (38)-(39) result in following transformations laws for the solutions of the system (40):

$$
\begin{gathered}
p \rightarrow p^{(1)}=\frac{A+\Omega\left(\psi_{1}, \phi_{1} ; p, f\right)}{\psi_{1}}, \\
f \rightarrow f^{(1)}=2 \alpha f+\frac{u}{\psi_{1}}\left(A+\Omega\left(\psi_{1}, \phi_{1} ; p, f\right)\right), \\
f \rightarrow{ }^{(1)} f=\frac{A+\Omega\left(\psi_{1}, \phi_{1} ; p, f\right)}{\phi_{1}}, \\
p \rightarrow{ }^{(1)} p=-2 \alpha p+\frac{v}{\phi_{1}}\left(A+\Omega\left(\psi_{1}, \phi_{1} ; p, f\right)\right),
\end{gathered}
$$

where

$$
\begin{aligned}
& \Omega(\psi, \phi ; p, f)=\int d \Omega(\psi, \phi ; p, f), \quad d \Omega(\psi, \phi ; p, f)=(\psi p+\phi f) d x \\
& +\alpha(\psi p-\phi f) d y+2 i\left[\frac{1}{\alpha}(v \psi f+u \phi p)+p \psi_{x}-p_{x} \psi+f_{x} \phi-f \phi_{x}\right] d t
\end{aligned}
$$

$A$ is arbitrary constant, 1 -form $d \Omega(\psi, \phi ; p, f)$ is closed if $\psi, \phi, p$ and $f$ are solutions of the (37), (40) with the same potentials $u, v$ and $F$. Similar formulas for $\psi_{(1)}$, $\phi_{(1)},{ }_{(1)} \psi,{ }_{(1)} \phi$ which are induced by the transformations (41)-(42) have the form:

$$
\begin{gathered}
\psi \rightarrow \psi_{(1)}=\frac{A+\Omega\left(\psi, \phi ; p_{1}, f_{1}\right)}{p_{1}}, \\
\phi \rightarrow \phi_{(1)}=-2 \alpha \phi+\frac{v}{p_{1}}\left(A+\Omega\left(\psi, \phi ; p_{1}, f_{1}\right)\right), \\
\phi \rightarrow{ }_{(1)} \phi=\frac{A+\Omega\left(\psi, \phi ; p_{1}, f_{1}\right)}{f_{1}}, \\
\psi \rightarrow{ }_{(1)} \psi=2 \alpha \psi+\frac{u}{f_{1}}\left(A+\Omega\left(\psi, \phi ; p_{1}, f_{1}\right)\right) .
\end{gathered}
$$


Using (38), (39), (41-44) one can find elemantary binary DT. These transformations allow us to calculate potentials with two indices: one on top and one below. For example

$$
u_{(1)}^{(1)}={ }_{(1)}^{(1)} u=\frac{1}{{ }_{(1)} v^{(1)}}=u+\frac{2 \alpha f_{1} \psi_{1}}{A+\Omega_{1}}, \quad v_{(1)}^{(1)}={ }_{(1)}^{(1)} v=\frac{1}{{ }_{(1)} u_{(1)}}=v-\frac{2 \alpha p_{1} \phi_{1}}{A+\Omega_{1}},
$$

where $\Omega_{1}=\Omega\left(\psi_{1}, \phi_{1} ; p_{1}, f_{1}\right)$. We'll not write out these formulas.

Remark. The twain of linear equations for the $\psi_{y}, \phi_{y}$ (37) (or for the $p_{y}, f_{y}$ (40)) can be represented as the single linear second-order equation with variable coefficients. Imposing the special restrictions on the potentials $u$, $v$, one can obtain some famous equations, e.g. Laplace-Moutard equation [11] or the Goursat one [12]. To obtain the Goursat equation one need to choose

$$
v= \pm u \text {. }
$$

The usual Darboux transformations don't conserve the reduction restrictions (46), while the elementary binary transformations (45) conserve them by the choice

$$
p=\psi, \quad f=\mp \phi,
$$

which don't conflict with (37), (40). As a result we get a well known analog of a Moutard transformation for the Gourst equation (see, for example, [13], [14), which is nothing but outcome of two successive elementary DT. Similarly it is possible to obtain usual Moutard transformation. Note that this transformation is the useful method to construct explicit solutions of the Veselov-Novikov equation [10], [15]. In turn the Goursat equation produce $2 D \mathrm{mKdV}$ hierarchy so transformations (45) (which don't conflict with (46)) can be used to construct exact solutions of equations from this hierarchy.

The DS equations (36) admits ST ([7], [16]):

$$
\begin{aligned}
& u_{n} \rightarrow u_{n+1}=u_{n}\left(u_{n} v_{n}+\alpha^{2}\left(\log u_{n}\right)_{x x}-\left(\log u_{n}\right)_{y y}\right), \quad v_{n} \rightarrow v_{n+1}=\frac{1}{u_{n}}, \\
& g_{n} \rightarrow g_{n+1}=g_{n}+4\left(\log u_{n}\right)_{x x} \\
& u_{n} \rightarrow u_{n-1}=\frac{1}{v_{n}}, \quad v_{n} \rightarrow v_{n-1}=v_{n}\left(u_{n} v_{n}+\alpha^{2}\left(\log v_{n}\right)_{x x}-\left(\log v_{n}\right)_{y y}\right), \\
& g_{n} \rightarrow g_{n-1}=g_{n}+4\left(\log v_{n}\right)_{x x},
\end{aligned}
$$

from which one get the equations of $2 D$ Toda lattice:

$$
\alpha^{2} q_{n, x x}-q_{n, y y}=e^{q_{n+1}-q_{n}}-e^{q_{n}-q_{n-1}}, \quad q_{n}=\log \left(u_{n}\right) .
$$

Using (48) and (36) we find symmetries of the (48) (see. (23):

$$
-i q_{n, t}=2 q_{n, x x}+q_{n, x}^{2}+\frac{1}{\alpha^{2}} q_{n, y}^{2}-\frac{1}{\alpha^{2}}\left(e^{q_{n+1}-q_{n}}+e^{q_{n}-q_{n-1}}\right)+g_{n},
$$

where $g_{n}$ can be expressed as:

$$
g_{n}(x, y, t)=\tilde{g}_{n}(x, y)+4 \int_{-\infty}^{\infty} d x^{\prime} d y^{\prime} G\left(x^{\prime}, y^{\prime} ; x, y\right)\left(e^{q_{n}-q_{n-1}}\right)_{x^{\prime} x^{\prime}},
$$

where $G\left(x^{\prime}, y^{\prime} ; x, y\right)$ is the Green function satisfying the equation

$$
\left(\partial_{y}^{2}-\alpha^{2} \partial_{x}^{2}\right) G\left(x^{\prime}, y^{\prime} ; x, y\right)=-\delta\left(x-x^{\prime}\right) \delta\left(y-y^{\prime}\right)
$$

where $\tilde{g_{n}}$ is the solution of this equation with a zero second member of this one. 
The plan of searching of Lax pair, Darboux transformations and dressing chains for the (48) is the same as the plan in Sec. 2: Using (37), (40) and (47) one find two "conjugate" Lax pairs for the (48):

$$
\begin{aligned}
& \psi_{n, y}=\alpha \psi_{n, x}+e^{q_{n}-q_{n-1}} \psi_{n-1}, \\
& \psi_{n+1}=2 \alpha \psi_{n, x}-\left(\alpha q_{n, x}+q_{n, y}\right) \psi_{n}+e^{q_{n}-q_{n-1}} \psi_{n-1},
\end{aligned}
$$

and

$$
\begin{aligned}
& f_{n, y}=-\alpha f_{n, x}-e^{q_{n}-q_{n-1}} f_{n-1} \\
& f_{n+1}=2 \alpha f_{n, x}+\left(q_{n, y}-\alpha q_{n, x}\right) f_{n}+e^{q_{n}-q_{n-1}} f_{n-1} .
\end{aligned}
$$

Using now DT (38-39) and (41-42) it is easy to obtain requisite formulas for the (49), (50). The result is just the same as $R$ - and $L$-transformations (14-15), and this is true for both the (49) and (50). The last statement is obvious because the Lax pair (50) can be obtained from the (49) by the substitute

$$
\alpha \rightarrow-\alpha, \quad \psi_{n+k} \rightarrow f_{n+k}=(-1)^{k} \psi_{n+k} .
$$

Starting out from the (49) one get $2 D$ Volterra equations (compare to (17)):

$$
\xi_{n, X Y}=\xi_{n, X}\left(e^{\xi_{n}-\xi_{n+1}}-e^{\xi_{n-1}-\xi_{n}}\right),
$$

where

$$
\partial_{X}=\partial_{y}+\alpha \partial_{x}, \quad \partial_{Y}=\partial_{y}-\alpha \partial_{x}, \quad \xi_{n, X}=\frac{\psi_{1, n+1}}{\psi_{1, n}}
$$

where $\psi_{1, n}$ is some particular solution of the (49). Introducing new dependent variable $a_{n}$ and $b_{n}$ :

$$
a_{n}=\xi_{n, x}, \quad b_{n}=e^{\xi_{n}-\xi_{n+1}},
$$

it is possible to present (51) in more customary form

$$
\left(\log a_{n}\right)_{Y}=b_{n}-b_{n-1}, \quad\left(\log b_{n}\right)_{X}=a_{n}-a_{n+1} .
$$

And If we start from the (50) then the resulting equations will be gage-equivalent to (51). But we'll not apply them here.

Finally, let apply dressing chains. Choosing Lax pair (49) and $R$-transformation (14) (it can be obtained from the (38)) we have:

$$
e^{\zeta_{n}-\zeta_{n+1}} \zeta_{n, X}=e^{\xi_{n}-\xi_{n+1}} \xi_{n+1, X}, \quad\left(\zeta_{n}-\xi_{n}\right)_{Y}=e^{\zeta_{n-1}-\zeta_{n}}-e^{\xi_{n}-\xi_{n+1}},
$$

where $\zeta_{n}$ is defined just as in Sec. 2 .

Chains (53) are $2 D$ generalization of chains (18). In addition , using binary DT we can construct dressing chains of the second type. Denoting (see (43))

$$
\eta_{n, Y}=\frac{f_{n+1}^{(1)}}{f_{n}^{(1)}}
$$

one get

$$
\begin{gathered}
e^{\eta_{n+1}-\eta_{n}} \eta_{n, Y}=e^{\xi_{n}-\xi_{n+1} \xi_{n+1, X},} \\
\left(\eta_{n,_{X}}-e^{\eta_{n}-\eta_{n-1}}\right)_{Y}=\left(e^{\xi_{n}-\xi_{n+1}}-\xi_{n, Y}\right)_{X} .
\end{gathered}
$$

Eliminating $\xi_{n}$ from the (54) we obtain nonlinear equation for the functions $\eta_{n}$, whereas chains (54) can be considered as Lax pair for this equation. 


\section{Conclusion}

Main results of this work are:

1. Dressing chains for the Toda lattice are constructed starting out from the NLS theory.

2. It is shown that dressing chains for the Toda lattice are closed.

3.The methods allowing to construct the $m_{k}^{n} T L$ equations are suggested. The particular example of equation from this set (including it's Lax pair, (33-34)) is studied.

4. Dressing chains of the first and second type for the $2 D$ Toda equations are obtained.

The links between NLS and TL were studied in [5]. Instead of traditional system of Zakharov-Shabat (3), authors of [5] have used the single second-order equation

$$
\Psi_{x x}+(z-2 i \lambda) \Psi_{x}+p \Psi=0,
$$

which can be obtained from the (3) if one put

$$
z=-(\log u)_{x}, \quad p=u v, \quad \Psi=e^{i \lambda x} \psi .
$$

Symmetries from the [5] are nothing but ST (6) (in [5] these transformations were called T-transformations) and DT (S-transformations in [5]). According to lemma which was proved in [5], S- and T-transformations are unique iso-spectral symmetries in the form $\Psi \rightarrow f \Psi_{x}+g \Psi$, where coefficients $f$ and $g$ are independent from the spectral parameter $\lambda$. It is obviously that transformations (4-5) and (11) don't satisfy this demand. One can show that they are connected with transformations $T^{-1}$ and $S^{-1}$. It is obvious in the case (4-5). The link between $S^{-1}$ and (11) has more delicate nature, so let consider it in detail.

The straightforward calculation by the (12) result in S-transformation for the equation (55):

$$
\Psi \rightarrow{ }^{(1)} \Psi=\Psi-\frac{\Psi_{x}}{\Psi_{1, x}} \Psi_{1},
$$

where $\Psi_{1}$ is the solution of the (55) with $\lambda=\lambda_{1}$.

Let us find the inverse transformation, acting accordingly to the [5]: Differentiating (56) and excluding $\Psi_{x}$ we get,

$$
\Psi=\Psi\left({ }^{(1)} \Psi,{ }^{(1)} \Psi_{x} ; \Psi_{1}, \Psi_{1, x}\right) .
$$

but it is not the ultimate result. That is because during the inversion of the formula (56) one must have in mind that second member (57) must be expressed via "dressed" values only (i.e. via values with left superscript "(1)"). To lead (57) to this form we need to introduce solution $\hat{\Psi}$ which will be linear-independent with $\Psi$. The general solution of (55) (with fixed $\lambda$ ) will be linear combination of $\Psi$ and $\hat{\Psi}$ with any given constant coefficients.

It is easy to find the function $\hat{\Psi}$ by the standard algorithm (to multiply (55) by the $\hat{\Psi}$; to multiply the equation for the $\hat{\Psi}$ by the $\Psi$; to subtract and to integrate). Then one can dress $\hat{\Psi}_{1}$ (for the $\lambda=\lambda_{1}$ ) by the formula (56) (we need to use this roundabout way to escape the result ${ }^{(1)} \Psi_{1}=0$, which we have faced by the direct application of the (57)):

$$
\hat{\Psi}_{1} \rightarrow^{(1)} \hat{\Psi}_{1}
$$


We differentiate this formula, then we express $\Psi_{1}$ and $\Psi_{1, x}$ via ${ }^{(1)} \hat{\Psi}_{1},{ }^{(1)} \hat{\Psi}_{1, x}$ and substitute it into the (57). Now it is possible to determine that (57) is gageequivalent to (11). Let $\psi$ and $\phi$ be solutions of the (3). The necessary for us linear-independent functions are:

$\hat{\psi}(x)=\frac{1}{2} \psi(x) \int d z \operatorname{sgn}(x-z) \frac{u(z)}{\psi^{2}(z)}, \quad \hat{\phi}(x)=\frac{1}{2} \phi(x) \int d z \operatorname{sgn}(x-z) \frac{u(z)}{\psi^{2}(z)}-\frac{i}{\psi}$.

Dressing these expessions by the formulas (12) and substituting $\lambda=\lambda_{1}, \psi=\psi_{1}$, $\phi=\phi_{1}$, one get

$$
{ }^{(1)} \hat{\psi}_{1}=\frac{i}{\phi_{1}}, \quad{ }^{(1)} \hat{\phi}_{1}=-\frac{i v}{\phi_{1}} .
$$

Finally, substituting obtained functions into the (11) we have

$$
{ }^{(1)} u \rightarrow-u, \quad{ }^{(1)} v \rightarrow-v
$$

Q.E.D.

The calibration (55) from the [5] is suitable to research closure conditions and for construction of potentials which are invariant with respect to $\mathrm{S}$ - and Ttransformations. In contrast to [5], our purpose here is the constructions of the dressing chains, in the manner of [8]. I conclude that the standard symmetrical calibration of Zakharov-Shabat is more comfortable and natural for this goal.

\section{Acknowledgements.}

The author are grateful to V. Yurov for useful comments and his help. This work was partially supported by the Grant of Education Department of the Russian Federation, No. E00-3.1-383.

\section{References}

[1] S.I. Svinolupov, R.I. Yamilov, Theor. Math. Phys. 98. no.2 (1994), 207.

[2] V.A. Adler, A.B. Shabat, Theor. Math. Phys. 111, no.3 (1997), 323.

[3] D. Levi, J. Phys. A: Math. Gen. 14 (1981) 1083; Also, A.B. Shabat, R.I. Yamilov, Algebra and analysis, vol.2, no.2 (1990), 183; A.N. Leznov, A.B. Shabat, R.I. Yamilov, Phys. Lett. A., vol.174 (1993), 397.

[4] R.I. Yamilov, Uspeh. Math. Nauk. 38, no.6 (1983), 155; Also V.A. Adler, A.B. Shabat, Theor. Math.Phys., vol.112, no.2 (1997), 179; V.A. Adler, A.B. Shabat, Theor. Math.Phys. vol.115, no.3 (1998), 349.

[5] A.B. Shabat, Theor. Math. Phys. 121, no.1 (1999), 165.

[6] A.V. Yurov, Theor. Math. Phys. 119, no.3 (1999), 419.

[7] A.V. Yurov, J. Math. Phys. 44, no.3 (2003), 1183.

[8] B.A. Borisov, S.A. Zykov, Theor. Math. Phys. 115, no.2 (1998), 199.

[9] S.B. Leble, N.V. Ustinov, J. Math. Phys. 34 (1993), 1421.

[10] V.B. Matveev, M.A. Salle, Darboux Transformation and Solitons, Berlin-Heidelberg: Springer Verlag, 1991.

[11] T. Moutard, J. Ecole Polytechnique 45 (1878), 1.

[12] E. Goursat, Bull. Soc. Math. France 25 (1897), 36.

[13] E.I. Ganzha, On One Analogue of the Moutard Transformation for Goursat Equation $\theta_{x y}=$ $2 \sqrt{\lambda(x, y) \theta_{x} \theta_{y}}$, solv-int @ xyz.lant.gov No 9806001 (1998).

[14] S.B. Leble, A.V. Yurov, J. Math. Phys. 43, no.2 (2002), 1095.

[15] C. Athorne, J.J. Nimmo, Inverse Problems 7 (1991), 809.

[16] A.V. Yurov, Theor. Math. Phys. 109, no.3 (1996), 338. 
Theoretical Physics Department, Kaliningrad State University, Kaliningrad, RusSIA

E-mail address: yurov@freemail.ru 